\title{
GENERALIZED FROBENIUS COMPLEMENTS
}

\author{
BETTY LOU AND D. S. PASSMAN
}

A good deal of work is being done at present on generalized Frobenius kernels, that is groups which admit a fixed point free automorphism of not necessarily prime order. In this paper we offer a candidate for the title "generalized Frobenius complement." In order to avoid the more difficult problem of studying the groups being acted upon, we consider here only linear groups. We will also assume throughout that the order of the groups are relatively prime to the characteristic of the field so that we have a completely reducible situation.

Definition. Let (5) be a linear group. Set $\theta$ (\$) equal to the normal subgroup of (5) generated by those elements which have a nonzero fixed point. We call $\$ / \theta(\$)$ a generalized Frobenius complement.

In $\$ 1$ we obtain a few simple results about these groups and in $\$ 2$ we show that these groups come up in a natural way in a problem of D. R. Hughes.

1. Basic properties. Let $\mathfrak{A}$ be a group of automorphisms of group $\mathfrak{B}$ which acts locally fixed point free, that is, each nonidentity element of $\mathfrak{A}$ acts fixed point free. Then by a theorem of John Thompson, $\mathfrak{B}$ is nilpotent and hence has a characteristic elementary abelian subgroup $\mathfrak{W}$ of order $p^{n}$ for some prime $p$. We can, of course, think of $\mathfrak{B}$ as a vector space of dimension $n$ over $G F(p)$. Clearly $\mathfrak{A}$ acts locally fixed point free on $\mathfrak{B}$. Since $A \in \mathfrak{A}$ has a nonzero fixed point in a vector space if and only if $A$ has an eigenvalue equal to 1 , we see that $\mathfrak{A}$ also acts locally fixed point free on $\mathfrak{W}^{*}=F \otimes \mathfrak{B}$ for any field $F \supseteq G F(p)$. Let $F$ be a splitting field for $\mathfrak{A}$. If $\mathfrak{W}_{1}$ is an absolutely irreducible constituent of $\mathfrak{W}^{*}$, then clearly $\mathfrak{A}$ acts locally fixed point free on $\mathfrak{W}_{1}$. Moreover, since the characteristic of $F$ is prime to $|\mathfrak{A}|, \mathfrak{W}_{1}$ comes from a complex representation of $\mathfrak{A}$. Thus every Frobenius complement has an irreducible complex representation in which it acts locally fixed point free.

The above does not hold for generalized Frobenius complements. Let $(B)$ act on vector space $\mathfrak{B}$. We see easily that $\theta(\mathbb{B})$ is invariant under field extension. Since the characteristic of the field is prime to $|(\$)|$ by assumption we see that we can view (B) as a complex linear group and $\theta(\$)$ remains the same. But now let $\mathfrak{W}_{1}$ be an irreducible constituent of $\mathfrak{B}$. Let $\Omega_{1}$ be the kernel of the action of $\mathbb{B}$ on $\mathfrak{W}_{1}$.

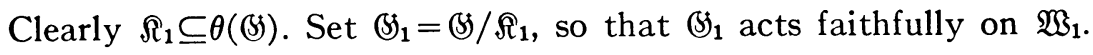

Received by the editors April 4, 1966. 
We see easily that $\theta(\$) / \Omega_{1} \supseteq \theta\left(\oiint_{1}\right)$ and the inclusion may be proper.

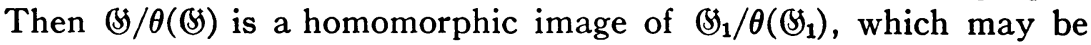
proper.

The following question is then pertinent. Given linear group (\$),

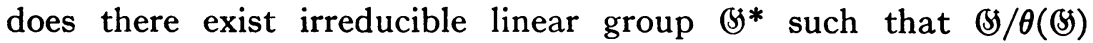
$\simeq \mathbb{S F}^{*} / \theta\left(\mathbb{S}^{*}\right)$ ? Unfortunately we have not been able to resolve this problem.

THEOREM 1. Let $\mathfrak{A}$ be a group which acts locally fixed point free. Let D be a central cyclic subgroup. Then there exists linear group (S) with (3) $/ \theta(\mathfrak{B}) \simeq \mathfrak{A} / \mathfrak{D}$.

Proof. Let $|\mathfrak{D}|=d$ and set $\mathfrak{B}=\mathfrak{H} \times \mathfrak{D}^{*}$ where $\mathfrak{D}^{*}=\left\langle D^{*}\right\rangle$ is cyclic of order $d$. If $\mathfrak{D}=\langle D\rangle$ set $\Im_{1}=\left\langle D^{*}\right\rangle$ and $\Im_{2}=\left\langle D D^{*}\right\rangle$. Then $\Im_{i}$ is cyclic of order $d, \mathfrak{D} \cap \Im_{i}=\langle 1\rangle$ and $\Im_{1} \Im_{2}=\mathfrak{D D}^{*}$. Since $\mathfrak{D}$ is central in $\mathfrak{A}$ we have $\mathfrak{Y}_{i} \triangle \mathcal{H}$ and $\mathbb{S} / \mathfrak{Y}_{i} \simeq \mathfrak{A}$. Let $\mathfrak{X}_{i}$ be an irreducible representation of (B) $/ \Im_{i}$, viewed as one of $B$, in which $B / \Im_{i}$ acts locally fixed point free. Since $\mathfrak{Y}_{1} \cap \mathfrak{Y}_{2}=\langle 1\rangle$ we see that $\mathfrak{X}=\mathfrak{X}_{1}+\mathfrak{X}_{2}$ is a faithful representation

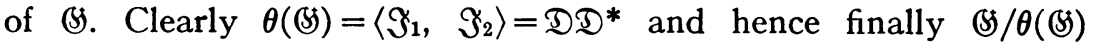
$=\mathfrak{U} \mathfrak{D}^{*} / \mathfrak{D D}^{*} \simeq \mathfrak{U} / \mathfrak{D}$.

If we let $\mathfrak{A}$ be the quaternion group of order $2^{n+1}$ then we see that $\mathfrak{A} / Z(\mathfrak{A})$, the dihedral group of order $2^{n}$, occurs as a reducible generalized Frobenius complement. If $n=2$ this group is abelian of type $(2,2)$. We now show that under the assumption of regularity such groups cannot occur.

Theorem 2. Let (S) be a linear group and suppose that all Sylow subgroups of $\$$ are regular. Then $\$ / \theta(\$)$ is a $Z$-group, that is a group with all Sylow subgroups cyclic.

Conversely, let $\&$ be any Z-group. Then there exists linear group (\$) having all Sylow subgroups regular with $\$ / \theta(\$) \simeq \mathbb{R}$.

Proof. Given $(S)$. Let $\mathfrak{B}$ be a Sylow $p$-subgroup of $(\mathbb{S}$ so that

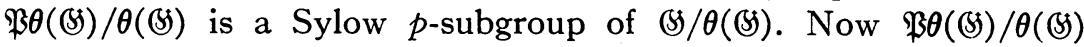
$\simeq \mathfrak{B} /(\mathfrak{B} \cap \theta(\mathfrak{H}))$ and clearly $\mathfrak{B} \cap \theta(\mathbb{S}) \supseteq \theta(\mathfrak{B})$. Hence a Sylow $p$-subgroup of $\mathbb{B} / \theta(\mathfrak{B})$ is a homomorphic image of $\mathfrak{B} / \theta(\mathfrak{B})$ and it suffices to show that $\mathfrak{B} / \theta(\mathfrak{B})$ is cyclic.

We show by induction on $|\mathfrak{P}|$ that if $\mathfrak{B}$ is a regular $p$-group which is a linear group then $\mathfrak{B} / \theta(\mathfrak{B})$ is cyclic. Let $\mathfrak{U}$ be a minimal counterexample. Suppose that $\mathfrak{B}$ acts on space $\mathfrak{B}$. Let $\mathfrak{U}$ be an irreducible submodule and let $\Re$ be the kernel of the representation of $\mathfrak{B}$ on $\mathfrak{U}$. Set $\mathfrak{P}_{2}=\mathfrak{B} / \mathfrak{\Omega}$ so that $\mathfrak{P} / \theta(\mathfrak{B})$ is a homomorphic image of $\mathfrak{P}_{2} / \theta\left(\mathfrak{P}_{2}\right)$. Since $\mathfrak{B}$ is a minimal counterexample we must have $\Re=\langle 1\rangle$. Thus we can assume that $\mathfrak{B}$ is irreducible by taking $\mathfrak{P}=\mathfrak{B}_{2}$. This implies that 
the center of $\mathfrak{B}$ is cyclic and acts fixed point free. Moreover, using the fact that a regular 2-group is abelian, we can assume that $\mathfrak{P} / \theta(\mathfrak{B})$ is abelian of type $(p, p)$.

If $\theta(\mathfrak{B})=\langle 1\rangle$ then $\mathfrak{P}$ acts locally fixed point free and is cyclic. Since this is not the case, $\mathfrak{S}=\theta(\mathfrak{B})$ is nontrivial. Let $Z$ be a central element of $\mathfrak{P}$ of order $p$ contained in $\mathfrak{S}$. Let $X \in \mathfrak{P}-\mathfrak{S}$ and set $\mathfrak{A}=\langle X, Z\rangle$ so that $\mathfrak{A}$ is abelian. Suppose that $X^{p}$ has a nonzero fixed point in $\mathfrak{B}$. Then $\mathfrak{A} /\left\langle X^{p}\right\rangle$ acts on $\mathfrak{B}$, the space of fixed points of $X^{p}$. This quotient group is clearly of type $(p, p)$ since $Z \notin\left\langle X^{p}\right\rangle$. Now a group of type $(p, p)$ cannot act locally fixed point free. But $Z$ acts fixed point free and $X Z^{i} \in \mathfrak{B}-\mathfrak{S}$ for all $i$. Hence this cannot occur and $X^{p}$ has no nonzero fixed points in $\mathfrak{W}$.

Set $\mathfrak{P}_{1}=\Omega^{1}(\mathfrak{B})$, the subgroup of $\mathfrak{B}$ generated by all $p$ th powers of elements of $\mathfrak{B}$, and set $\mathfrak{S}_{1}=\Omega^{1}(\mathfrak{S})$. We have $\mathfrak{P}_{1} \supseteq \mathfrak{S}_{1}$ and by properties of regular $p$-groups and the above results, if $X \in \mathfrak{P}_{1}-\mathfrak{S}_{1}$ then $X$ has no fixed points in $\mathfrak{W}$. Hence $\theta\left(\mathfrak{B}_{1}\right) \subseteq \mathfrak{S}_{1}$. Consider the map $\alpha: \mathfrak{B} \rightarrow \mathfrak{P}_{1} / \mathfrak{W}_{1}$ given by $\alpha(X)=X^{p} \mathfrak{S}_{1}$. Since $\mathfrak{S} \supseteq \mathfrak{P}^{\prime}$ we see by regularity that $\alpha$ is a homomorphism of $\mathfrak{P}$ onto $\mathfrak{P}_{1} / \mathfrak{W}_{1}$. The kernel clearly contains $\mathfrak{S}$. If $X$ is in the kernel then $X^{p}=H^{p}$ for some $H \in \mathfrak{S}$. By regularity $\left(X H^{-1}\right)^{p}=1$ and thus $\left(X H^{-1}\right)$ is an element whose $p$ th power has fixed points. Hence $X H^{-1} \in \mathfrak{S}$ and $X \in \mathfrak{S}$. Therefore $\mathfrak{S}$ is the kernel of $\alpha$ and $\mathfrak{P} / \mathfrak{S}_{2} \simeq \mathfrak{P}_{1} / \mathfrak{S}_{1}$. Since $\mathfrak{B}_{1}<\mathfrak{P}$ and $\theta\left(\mathfrak{P}_{1}\right) \subseteq \mathfrak{S}_{1}$ we have $\mathfrak{P}_{1} / \theta\left(\mathfrak{P}_{1}\right)$ is cyclic and hence $\mathfrak{P}_{1} / \mathfrak{S}_{1}$ is cyclic, a contradiction. This proves the result.

Now let $\mathfrak{R}$ be a $Z$-group. Then $\mathfrak{R}=\mathfrak{A} \mathfrak{B}$ where $\mathfrak{A}$ and $\mathfrak{B}$ are cyclic of relatively prime order and $\mathfrak{A} \triangle \mathfrak{R}$. Let $|\mathfrak{B}|=b$ and set $\mathfrak{B}^{*}=\langle B\rangle$ where $B$ has order $b^{2}$. If $\mathfrak{D} \subseteq \mathfrak{B}^{*}$ with $\mathfrak{D}=\left\langle B^{b}\right\rangle$ then $\mathfrak{D}$ is cyclic of order $b$ and $\mathfrak{B}^{*} / \mathfrak{D} \simeq \mathfrak{B}$. Let $\mathfrak{A}^{*}=\mathfrak{H} \times{ }_{\sigma} \mathfrak{B}^{*}$, the semidirect product of $\mathfrak{A}$ by $\mathfrak{B}^{*}$ where $\mathfrak{B}^{*}$ acts on $\mathfrak{A}$ by way of the isomorphism $\mathfrak{B}^{*} / \mathfrak{D} \simeq \mathfrak{B}$. Thus $\mathfrak{D}$ is a central subgroup of $\mathfrak{U}^{*}$ and $\mathfrak{A}^{*} / \mathfrak{D} \simeq \mathfrak{A} \mathfrak{B}=\mathfrak{R}$. Now $\mathfrak{Y} \mathfrak{D}$ is a normal cyclic subgroup of $\mathfrak{2}^{*}$ and thus $\mathfrak{A}^{*}$ has an irreducible representation $\mathfrak{X}$ with every element of $\mathfrak{A} \mathfrak{D}$ acting fixed point free. Also if $X \in \mathfrak{A}^{*}$ and $X \neq 1$, then some power of $X$ is clearly a nonidentity element of $\mathfrak{A D}$. Hence $\mathfrak{A}^{*}$ acts locally fixed point free. By Theorem 1 there exists linear group (5) with $(\mathbb{S} / \theta(\mathbb{B}) \simeq \mathfrak{Q} * / \mathfrak{D} \simeq \mathbb{R}$. Since the group (\$) constructed has abelian Sylow subgroups the result follows.

The previous constructions all yield reducible linear groups. We now modify the methods used to obtain irreducible groups. However, we can no longer guarantee that the Sylow 2-subgroup of $(B)$ is regular.

THEOREM 3. Let $\mathfrak{L}=\mathfrak{A} \mathfrak{B}$ where $\mathfrak{A} \triangle \mathfrak{R}$ and $|\mathfrak{A}|$ and $|\mathfrak{B}|$ are rela- 
tively prime. Suppose that $\mathfrak{A}$ acts locally fixed point free and that $\mathfrak{B}$ is cyclic. Then there exists irreducible linear group $\mathbb{B}$ with $\mathbb{(} / \theta(\mathbb{B}) \simeq R$.

Proof. Let $p$ denote a prime. We define the following groups:

$$
\Re(2)=g p\left\langle R, S \mid R^{8}=S^{2}=1, S^{-1} R S=R^{3}\right\rangle .
$$

If $p^{a}>2$ then

$$
\Re\left(p^{a}\right)=g p\left\langle R, S \mid R^{p^{a+1}}=S^{p}=1, S^{-1} R S=R^{1+p^{a}}\right\rangle .
$$

If $m=\prod p_{i}^{a_{i}}$ set

$$
\Omega(m)=\prod \Omega\left(p_{i}^{a_{i}}\right) \text {, the direct product of these groups. }
$$

Lemma. Let $m=\prod p_{i}^{a_{i}}$ and set $m^{\prime}=\prod p_{i}$. Then $\Omega(m)$ contains subgroups $\mathfrak{D}$ and $\mathfrak{D}^{*}$ such that

(i) $\Re(m) \supseteq \mathfrak{D} \supseteq \mathfrak{D}^{*}$,

(ii) $\mathfrak{D} \triangle \mathfrak{\Omega}(m)$ and $\Re(m) / \mathfrak{D}$ is cyclic of order $m$.

(iii) $\mathfrak{D}^{*}=\mathbb{B} \times \mathbb{E}$ where 3 and $\mathbb{E}$ are cyclic subgroups of order $m^{\prime}$, 3 is central and $\left(^{\Omega(m)}=\mathfrak{D}\right.$.

(iv) If $X \in \Re(m)-\mathfrak{D}$, then some power of $X$ is a nonidentity element of 3 .

Proof. Since the groups $\Omega\left(p_{i}^{a_{i}}\right)$ have relatively prime orders, every subgroup of $\Omega(m)$ is the direct product of its projections on the $\Omega\left(p_{i}^{a_{i}}\right)$. Moreover, if $X \in \Omega(m)$ then the projection of $X$ on $\Omega\left(p_{i}^{a_{i}}\right)$ is some power of $X$. With this we see that it suffices to prove the result in the special case $m=p^{a}$.

First assume $p^{a}>2$ so that $\Omega=\Re\left(p^{a}\right)$ is generated by $R$ and $S$ with $R^{p^{a+1}}=S^{p}=1$ and $S^{-1} R S=R^{1+p^{a}}$. We see immediately that $Z(\Omega)$ $=\left\langle R^{p}\right\rangle$ and $\mathscr{R}^{\prime}=\left\langle R^{p^{a}}\right\rangle$. Set $\mathfrak{D}=\mathfrak{D}^{*}=\left\langle R^{p^{a}}, S\right\rangle$ with $\mathbb{B}=\left\langle R^{p^{a}}\right\rangle$ and $\mathfrak{E}=\langle S\rangle$. Now $\mathbb{Z}$ is central and since $\mathfrak{D} \supseteq \Omega^{\prime}, \mathfrak{D}$ is normal. Clearly $\mathfrak{\Omega} / \mathfrak{D}$ is cyclic of order $p^{a}$. Now $\mathbb{E}$ is not normal so $\mathfrak{D} \supseteq \mathbb{F}^{\Re} \supset \mathbb{E}$. Since $|\mathfrak{D}|=p^{2},|\mathfrak{E}|=p$ we see that $\mathbb{F}^{\mathfrak{R}}=\mathfrak{D}$. This proves (i), (ii) and (iii).

We show now that $\mathfrak{D}=\left\{X \in \Omega \mid X^{p}=1\right\}$. Since $\mathfrak{D}=\mathfrak{B} \times \mathfrak{E}$, $\mathfrak{D}$ has period $p$. Conversely, let $X^{p}=1$. Then $X \Omega^{\prime}$ has order 1 or $p$ in abelian group $\Omega / \Omega^{\prime}$. Since $\Omega / \Omega^{\prime}$ is abelian of type $\left(p^{a}, p\right)$ we see that $X \Omega^{\prime} \in\left\langle R^{p^{a-1}}, S\right\rangle \Omega^{\prime}$ and $X \in\left\langle R^{p^{a-1}}, S\right\rangle$. If $a \geqq 2$ then $\left\langle R^{p^{a-1}}, S\right\rangle$ is abelian of type $\left(p^{2}, p\right)$ and thus $X \in\left\langle R^{p^{a}}, S\right\rangle=\mathfrak{D}$ in this case. Now let $a=1$ so that $p=p^{a}>2$. Then $\Omega$ has order $p^{3}$ and it is well known that for $p$ odd the set of elements of $\Re$ of order 1 and $p$ is a subgroup. Since $\Omega$ does not have period $p$ and $\mathfrak{D}$ is a maximal subgroup in this case, the result follows. We show now that (iv) is satisfied. Let $X \notin \mathfrak{D}$ so that $X^{p} \neq 1$. Since $\langle R\rangle$ is a normal subgroup of $\Omega$ of index $p, X^{p}$ is 
a nonidentity element of $\langle R\rangle$. Then certainly some power of $X$ is equal to $R^{p^{a}}$.

Now let $p^{a}=2$ so that $\Omega=\Re(2)$ is generated by $R$ and $S$ with $R^{8}=S^{2}=1$ and $S^{-1} R S=R^{3}$. Set $\mathfrak{D}=\left\langle R^{2}, S\right\rangle$ and $\mathfrak{D}^{*}=\left\langle R^{4}, S\right\rangle$ with $\mathbb{Z}=\left\langle R^{4}\right\rangle$ and $\mathbb{E}=\langle S\rangle$. Then $\mathfrak{D}^{*}=\mathbb{B} \times \mathbb{E}, \mathbb{Z}$ is central and $\mathfrak{D}$ is normal. Thus $\mathfrak{D} \supseteq \mathscr{F}^{\Re}$. Since $\mathbb{F}^{\Re}$ contains $S$ and $R^{-1} S^{-1} R S=R^{2}$ we have $\left(^{\Re}=\mathfrak{D}\right.$ and (i), (ii) and (iii) are proved. Let $X \in \mathfrak{D}$. Then $X=R^{i}$ or $R^{i} S$ with $i$ odd. If $X=R^{i}$ then $X^{4}=R^{4}$ and if $X=R^{i} S$ then $X^{2}=R^{4}$. Thus the result follows.

We now return to the proof of the theorem. Let $b=|\mathfrak{B}|$ and let $\Re=\Re(b)$. Set $\mathfrak{S}=\mathfrak{A} \times_{\sigma} \Omega$, the semidirect product of $\mathfrak{A}$ by $\Omega$, where $\Re$ acts on $\mathfrak{A}$ by way of the isomorphism $\mathfrak{\Omega} / \mathfrak{D} \simeq \mathfrak{B}$.

Now (5) contains the subgroup $\mathfrak{A D}^{*}=\mathfrak{A} \times \mathfrak{B} \times \mathbb{E}$. Moreover, $(|\mathfrak{A}|,|\mathfrak{Z}|)=1, \mathfrak{Z}$ is cyclic and $\mathfrak{A}$ acts locally fixed point free. Hence there exists an irreducible complex representation $\mathfrak{Y}$ of $\mathfrak{A} \mathfrak{D}^{*}$ with kernel $\mathbb{E}$ and with $\mathfrak{A} \mathfrak{B}$ acting locally fixed point free. Let $\mathfrak{X}$ be an irreducible constituent of the induced representation of $\mathfrak{V}$ to (3). Since $\mathfrak{A} \mathbb{B} \triangle \mathfrak{G}$ we see that $\mathfrak{A} \mathbb{Z}$ acts locally fixed point free in the representation $\mathfrak{X}$. Let $X \in\left(S-\mathfrak{D}\right.$. We show that some power of $X$ is in $(\mathfrak{A} \mathscr{Z})^{\#}$, the set of nonidentity elements of $\mathfrak{A} \mathfrak{B}$. Suppose first that $X \notin \mathfrak{A D}$. Then since $B / \mathfrak{A} \simeq \Omega$ we conclude by the lemma that some power of

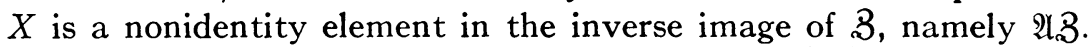
On the other hand, if $X \in \mathfrak{A} \mathfrak{D}=\mathfrak{A} \times \mathfrak{D}$ then since $(|\mathfrak{A}|,|\mathfrak{D}|)=1$ and $X \notin \mathfrak{D}$ some power of $X$ is in $\mathfrak{A}^{\#}$. Since $\mathfrak{A} \mathbb{Z}$ acts locally fixed point free, this implies that for all $X \in(B-D), X$ acts fixed point free.

Clearly $\theta(\mathfrak{S}) \subseteq \mathfrak{D}$. Since $\mathbb{E}$ has fixed points $\theta(\mathbb{S}) \supseteq \mathfrak{F}^{\mathfrak{B}}=\mathfrak{F}^{\mathfrak{Q}}=\mathfrak{I}$. Hence $\theta(\mathbb{S})=\mathfrak{D}$ and $\mathbb{S} / \theta(\mathbb{S})=\mathbb{S} / \mathfrak{D} \simeq \mathfrak{R} \mathscr{B}=\mathbb{R}$. This completes the proof.

Thus we see that all $Z$-groups and many groups like the semidirect product of the quaternion group of order 8 by a group of order 3 occur as irreducible generalized Frobenius complements. Now many of these groups are Frobenius groups which do not act locally fixed point free. Thus in some sense the generalized case is more satisfactory. We do not know whether any dihedral group can occur as an irreducible generalized Frobenius complement.

For any group $\mathfrak{A}$ let $\Omega_{1}(\mathfrak{A})$ denote the subgroup of $\mathfrak{A}$ generated by elements of order $p$ for all primes $p|| \mathfrak{A} \mid$.

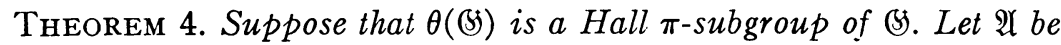
a $\pi$-complement in $\mathbb{S}$. Then $\mathfrak{A}$ acts locally fixed point free. If the Sylow 2-subgroup of $\theta(\mathfrak{B})$ is abelian, then $\Omega_{1}(\mathfrak{H})$ centralizes $\theta(\$)$. In any case $\Omega_{1}\left(\mathfrak{I} \mathfrak{I}^{\prime}\right)$ centralizes $\theta(\mathfrak{S})$. 
Proof. By the Schur-Zassenhaus theorem a $\pi$-complement $\mathfrak{A}$ exists. Since $\mathfrak{A} \cap \theta(\mathfrak{S})=\langle 1\rangle$ we see that $\mathfrak{A}$ acts locally fixed point free. Let $\mathfrak{P}=\langle P\rangle$ be a subgroup of $\mathfrak{A}$ of order $p$ and suppose that $\mathfrak{P}$ does not centralize $\mathfrak{S}=\theta(\mathfrak{S})$. We consider the group $\mathfrak{S P}$. By a result of Shult (Corollary 3.2 of [2]) $P$ has an eigenvalue equal to 1 unless $p$ is a Fermat prime and a Sylow 2 -subgroup of $\mathfrak{S}$ is nonabelian. Since $P \notin \theta(\$)$ we must have the latter two conditions holding. Since 2|| $\mathfrak{S} \mid$ we see that $\mathfrak{A}$ has odd order. Let $\mathfrak{B}^{*}$ be a Sylow $p$-subgroup of 2. Then $\mathfrak{P}^{*}$ is cyclic and since $p$ is a Fermat prime, $\mathfrak{P}^{*}$ is in the center of its normalizer in $\mathfrak{A}$. Hence by Burnside's theorem, $\mathfrak{A}$ has a normal $p$-complement. Since $\mathfrak{B}^{*}$ is abelian we see that $\mathfrak{B} \cap \mathfrak{A}^{\prime}=\langle 1\rangle$. Thus the result follows.

\section{Hughes' problems.}

DEFINITION. Let (\$) be a finite group. Let $H_{n}(\$)$ be the subgroup of (5) generated by all elements $G$ whose order does not divide $n$.

The general problem [1] is to study the quotient $(\$) / H_{n}(\mathbb{S})$ when $H_{n}(\$) \neq\langle 1\rangle$. We consider the special case in which there is a prime $g$ which divides $\mid$ (S) $\mid$ but $q \nmid n$.

THEOREM 5. Let $q \| \mid(S \mid$ but $q \nmid n$. Then there exists an irreducible linear group $\Re$ involved in (S) with $(\$) / H_{n}(\mathbb{S})$ a homomorphic image of $\Re / \theta(\Re)$.

Conversely let $\Re$ be any linear group. Then there exists group (S) and integer $n$ with $(B) / H_{n}(\$) \simeq \Re / \theta(\Re)$.

Proof. Let $\mathfrak{D}$ be a Sylow $q$-subgroup of $\left(\mathfrak{S}\right.$. Clearly $\mathfrak{D} \subseteq \mathfrak{S}=H_{n}(\mathfrak{S})$ since $q \nmid n$. Since $\mathfrak{S}$ is normal in $(B)$ we have $\mathfrak{B}=\mathfrak{S} N(\mathfrak{D})$ and thus (S) $/ \mathfrak{S} \simeq N(\mathfrak{D}) /\{\boldsymbol{N}(\mathfrak{D}) \cap \mathfrak{S}\}$. Now $H_{n}(\boldsymbol{N}(\mathfrak{D})) \subseteq \mathfrak{S} \cap N(\mathfrak{D})$ and therefore it suffices to assume that $\mathfrak{D}$ is normal in $\mathbb{B}$. By the Schur-Zassenhaus theorem $\mathfrak{D}$ has a complement $\mathbb{S}$ in $(\mathbb{S}$.

We study the action of $\mathfrak{S}$ on $\mathfrak{D}$. Let $\mathfrak{S}_{1}=\mathfrak{S} \cap \mathfrak{S}$. Since $\mathfrak{D} \subseteq \mathfrak{S}$ we see that $\mathfrak{H} / \mathfrak{S} \simeq \mathscr{S} / \mathfrak{S}_{1}$. Let $S \in \mathfrak{S}-\mathfrak{S}_{1}$. If $Q$ is any element of $\mathfrak{D}^{\#}$ then since $Q \in \mathfrak{S}, S \notin \mathfrak{S}$ we have $Q S \notin \mathfrak{S}$ and thus in particular $q$ does not divide the order of $Q S$. Hence $S$ does not centralize $Q$. Let $\mathfrak{B}$ be a characteristic elementary abelian subgroup of $\mathfrak{D}$. Then $\subseteq$ acts on $\mathfrak{B}$ in such a way that if $S \in \subseteq-\Im_{1}$ then $S$ acts fixed point free. Let $\mathfrak{B}$ be an irreducible $\subseteq$ subspace of $\mathfrak{W}$ and let $\Re$ be the kernel of the action $\subseteq$ on $\mathfrak{B}$. Set $\Re=\subseteq / \Re$. Clearly $\Re \subseteq \Im_{1}$ and $\theta(\Re) \subseteq \Im_{1} / \Re$. Hence $\subseteq / \mathfrak{S}_{1}$ is a homomorphic image of $\Re / \theta(\Re)$ and the result follows.

Conversely let us be given $\Re$. Choose prime $q \nmid|\Re|$ and let $\mathfrak{D}$ be an elementary abelian subgroup of suitable size so that the given representation of $\Re$ is realizable in $\mathfrak{D}$. Set $\mathfrak{S}=\mathfrak{D} \times{ }_{0} \Re$ and let $n$ be the 
period of $\mathfrak{R}$. We show that $H_{n}(\mathfrak{B})=\mathfrak{D} \theta(\Re)$. Clearly $H_{n}(\mathfrak{S}) \supseteq \mathfrak{D}$. Let $R \in \theta(\Re)$ be an element having a nonidentity fixed point $Q \in \mathfrak{D}$. Then

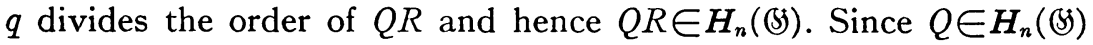

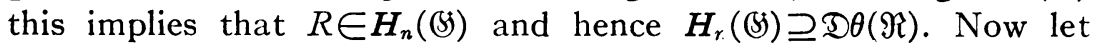
$Q \in \mathfrak{D}, R \in \Re$ such that the order of $Q R$ does not divide $n$. Then

$$
\begin{aligned}
1 \neq(Q R)^{n} & =Q^{1+R+\cdots+R^{n-1}} R^{n} \\
& =Q^{1+R+\cdots+R^{n-1}}=\widetilde{Q}
\end{aligned}
$$

since $R^{n}=1$ by definition of $n$. Now

$$
\widetilde{Q}^{(R-1)}=Q^{\left(1+R+\cdots+R^{n-1}\right)(R-1)}=Q^{R^{n}-1}=1
$$

so $\widetilde{Q}$ is a nonidentity fixed point in $\mathscr{D}$ of $R$ and hence $R \in \theta(\Re)$. This yields $H_{n}(\mathfrak{S}) \subseteq \mathfrak{D} \theta(\Re)$ and thus finally $\left(\mathbb{H} / H_{n}(\mathfrak{H})=\mathfrak{D} \Re / \mathfrak{D} \theta(\Re)\right.$ $\simeq \Re / \theta(\Re)$.

We can combine the above with the results of the first section to describe $\$ / H_{n}(\$)$ in certain cases. Since this process is straight forward we omit it.

\section{REFERENCES}

1. D. R. Hughes, Bull. Amer. Math. Soc. 63 (1957), 209.

2. E. E. Shult, On groups admitting fixed point free abelian operator groups, Illinois J. Math. 9 (1965), 701-720.

University of CaLIFornia, Los Angeles 